\title{
THEORETICAL MODEL AND DESIGN OF A NOVEL STATIC POLARIZATION WIND IMAGING INTERFEROMETER (NSPWII)
}

\author{
Chunmin Zhang ${ }^{1,2, *}$ Tingyu Yan ${ }^{1,2}$, Tingkui Mu ${ }^{1,2}$, Yifan $\mathrm{He}^{1,2}$ \\ ${ }^{1}$ Institute of Space Optics, School of Science, Xi' an Jiaotong University, \\ Xi’an, Shaanxi 710049, China - zcm@xjtu.edu.cn, yantingyu2010@qq.com, tkmu@xjtu.edu.cn, 825338471@qq.com \\ ${ }^{2}$ Key Laboratory for Nonequilibrium Synthesis and Modulation of Condensed Matter, \\ Xi'an, Shaanxi 710049, China
}

KEY WORDS: Polarization, Wind Imaging Interferometer, Field Widening, Jones Matrix, Atmosphere, Instrument

\begin{abstract}
:
Polarization array based polarization Michelson wind field detection interferometer is a new type of interferometer for detecting atmospheric temperature and wind field velocity. We proposed a theoretical model and design of the novel static polarization wind imaging interferometer (NSPWII). It consists of a group of polarizers and waveplates, a polarization beamsplitter, a field widened Michelson interferometer, a pyramid prism, and a polarization array. Based on the principle of polarization interference, a polarization array composed of four polarizers with $45^{\circ}$ polarization directions differences is directly in front of the detector, and four modulated light beams from the pyramid prism pass through the four polarizers. Then, interferograms with four different intensity are imaged on the detector simultaneously, which further provides the atmospheric temperature and wind field velocity. The advantages of this instrument are static (no moving parts), achromatic, and temperature compensated. It is capable of measuring the upper atmospheric wind field in real time with a high precision.
\end{abstract}

\section{INTRODUCTION}

The Earth's atmosphere is a natural barrier to protect the life on Earth and plays an important role in maintaining the climate stability of the Earth. Understanding of the Earth's atmosphere is the cornerstone of saving the global environment. In recent years, due to a variety of reasons, the Earth's atmosphere has been damaged, causing a series of problems such as the hole in the ozone layer and global climate change. Solving these problems first requires high-precision, large-scale atmospheric dynamic detection. Wind is the main carrier of atmospheric energy and momentum. Wind field and atmospheric temperature are important parameters of atmospheric dynamics. The measurement of the temperature, velocity and pressure of the upper atmospheric wind field will provide a large amount of data for the research of atmospheric physics and geophysics, medium and long-term weather forecasting, aerospace, and coping with global climate change.

The passive detection of the upper atmospheric wind field is based on the interference imaging spectroscopy and the Doppler effect of electromagnetic waves. It uses the airglow (aurora) in the upper atmosphere as the detection source, and detects the modulation of interference fringes using the interferometer with a large optical path difference. By measuring the Doppler shift of the wavelength of the emission lines, the temperature, velocity and other information of the upper atmosphere can be retrieved using the four-point algorithm. Interferometric atmospheric wind field detection has a good sensitivity, approximately $5 \mathrm{~m} / \mathrm{s}$. It does not need to bring additional light source, which means it is more suitable for space borne detection. Thus it has become a research focus in recent years.

Michelson interferometer can be used for passive detection of atmospheric wind field. According to the different ways

\footnotetext{
* Corresponding author
}

of obtaining the interference pattern, it can be divided into three types, the detection system of the moving mirror scanning mode, the detection system of the four-zone coating mode, and the detection system based on the polarization Michelson interferometer. Shepherd et al. proposed the principle of wide-angle Michelson interferometer (WAMI) (Shepherd, 2002). Subsequently, a series of detection systems with moving mirror scanning modes were developed, such as the wide-angle Michelson Doppler imaging interferometer (WAMDII) (Shepherd et al., 1985), the Wind imaging interferometer (WINDII) flew on the UARS satellite from 1991 to 2005 (Shepherd et al., 2012), the infrared Doppler Michelson interferometer for measuring stratospheric winds (SWIFT) (Gault et al., 2001), the E-Region wind interferometer (ERWIN) (Kristoffersen et al., 2013), as well as the ground based Michelson interferometer for airglow dynamics imaging (MIADI) (Langille et al., 2013). They require precise moving mirror scanning and cannot accurately detect rapidly changing targets. The four-zone coating mode system, such as the mesospheric imaging Michelson interferometer (MIMI) (Babcock, 2006), generate four phase steps $0^{\circ}, 90^{\circ}, 180^{\circ}$, and $270^{\circ}$ by coating four different multilayer dielectric films on the four zones of the mirror of one arm of the field widened Michelson interferometer. It can get four interferograms of the target at the same time to realize realtime detection. This mode has very high requirements for the performance of multi-layer dielectric films and is difficult to manufacture. The polarization Michelson wind imaging interferometer system uses a polarization interferometer (Ramsey et al., 1980). By rotating the polarizers, four interference intensities of different phases of the target light source are obtained. The representative instrument is polarizing atmospheric Michelson interferometer (PAMI), which also contains moving components and cannot realize real time measurement (Bird et al., 1995).

In this paper, we propose a novel static polarization wind imaging interferometer (NSPWII). It uses a combination of a pyr- 


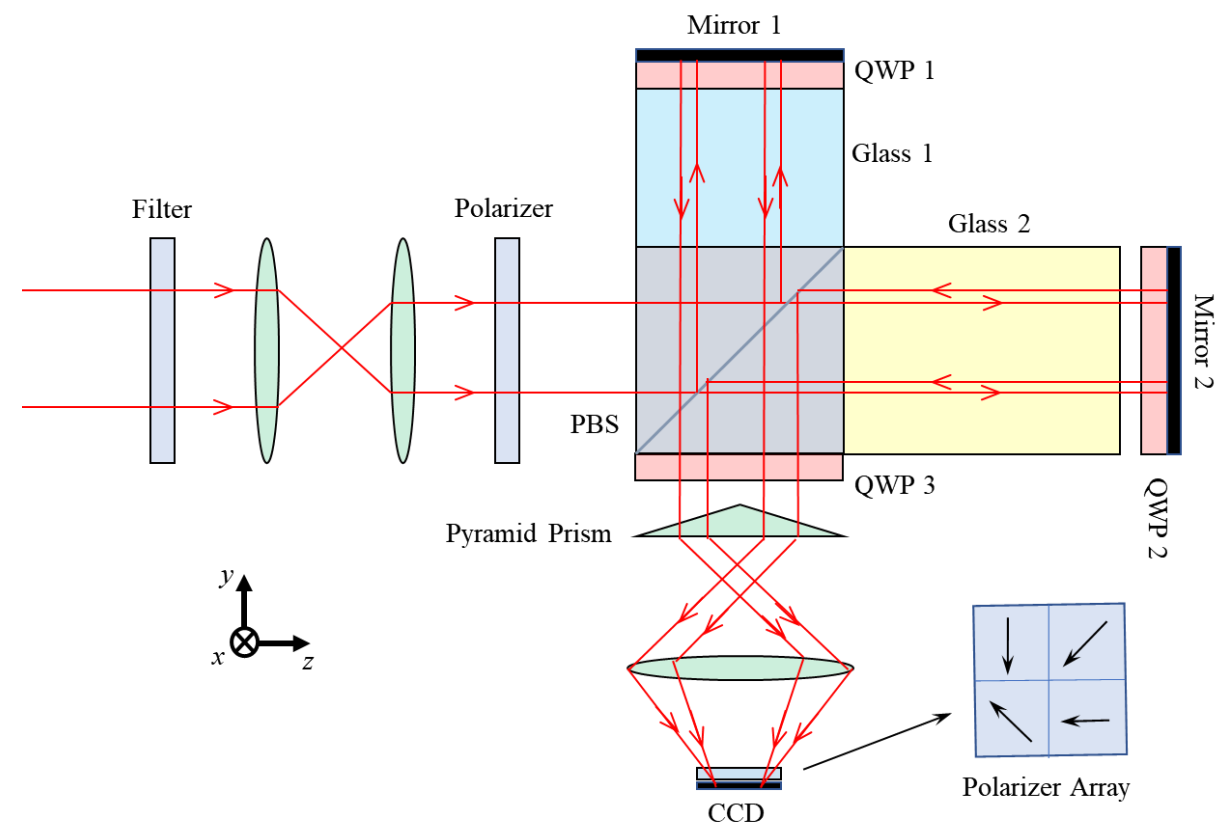

Figure 1. The system structure scheme of NSPWII

amid prism and a polarizing array instead of sequentially obtaining four interference intensities by rotating the polarizer four times in PAMI. It can obtain four interferograms with different phases on the four partitions of the detector simultaneously. Therefore, NSPWII can realize real time detection of targets, and the defect that the moving mirror scanning mode cannot accurately detect fast-changing targets has been overcome.

\section{PRINCIPLE}

The structure of NSPWII is shown in Fig. 1. It is mainly composed of a filter, a fore-optics system, a field widened polarization Michelson interferometer system, and imaging system. The function of the filter is to select the wavelength of the incident light wave and let the target wavelength that the instrument needs to detect pass through. The fore-optics system allows as much light as possible to enter the interferometer system, increasing the luminous flux of the system. The function of the pyramid prism is to make a beam of light equally divided into four beams in four different directions, and then the four beams are focused on the CCD through a imaging lens and a polarization array.

The field widened polarization Michelson interferometer is the core part of NSPWII. It consists of a polarizer, a polarization beamsplitter (PBS), two glass arms for field widening, three quarter wave plates (QWP), and two total reflection mirrors. Among them, the azimuth of the polarizer (specified as the angle between the transmission direction and the $x$ axis) and the azimuth of the three quarter-wave plates (specified as the angle between the fast axis and the $x$ axis) are $3 \pi / 4$. Passing through the optical filter, light at the specific wavelength is collimated by the fore-optics system, and incident into the polarizer. The exit light is linearly polarized with an azimuth of $3 \pi / 4$. The linearly polarized light can be decomposed into two components perpendicular to each other, $s$ component (the vibration direction is perpendicular to the paper surface) and $p$ component (the vibration direction is parallel to the paper surface). Assume that the light vector of the incident light wave passing through the filter, fore-optics, and polarizer is E, the two components of the light vector can be expressed by

$$
\begin{aligned}
& \mathrm{E}_{s}=A \cos \left(\frac{3 \pi}{4}\right) \exp \left[-i\left(\omega t-\mathbf{k} \cdot \mathbf{r}_{s}\right)\right] \\
& \mathrm{E}_{p}=A \cos \left(\frac{3 \pi}{4}\right) \exp \left[-i\left(\omega t-\mathbf{k} \cdot \mathbf{r}_{p}\right)\right]
\end{aligned}
$$

where $A$ is the amplitude, $\omega t$ is the initial phase and $\mathbf{k}$ is the wave vector of the incident light. $\mathbf{r}_{s}$ and $\mathbf{r}_{s}$ represent the position vectors of the $s$ and $p$ components of the light wave, respectively. After the light wave passes through the polarization beamsplitter PBS, its $s$ component is reflected and transmitted into arm 1, and the $p$ component is transmitted into arm 2. Since both ends of the arms have a 1/4 wave plate and a total reflection mirror, the path of transmitting light waves through the $1 / 4$ wave plate and then reflecting through the $1 / 4$ wave plate is equivalent to the light wave passing through a $1 / 2$ wave plate. The $s$ light of arm 1 will turn into $p$ light when returning and pass through the PBS without loss (assuming the performance of PBS is ideal). Its light vector components $\mathbf{E}_{1}{ }^{\prime}$ in the fast axis direction and slow axis direction of the wave plate are

$$
\begin{aligned}
& \mathbf{E}_{1 f^{\prime}}=A \cos \left(\frac{3 \pi}{4}\right) \exp \left[-i\left(\omega t-\mathbf{k} \cdot \mathbf{r}_{1}\right)\right] \cos \left(\frac{3 \pi}{4}\right) \\
& \mathbf{E}_{1 s}{ }^{\prime}=A \cos \left(\frac{3 \pi}{4}\right) \exp \left[-i\left(\omega t-\mathbf{k} \cdot \mathbf{r}_{1}\right)\right] \sin \left(\frac{3 \pi}{4}\right)
\end{aligned}
$$

Similarly, the $p$ light of arm 2 will turn into $s$ light when returning and reflecting by the PBS without loss. Its light vector components $\mathbf{E}_{2}{ }^{\prime}$ in the fast axis direction and slow axis direction of the wave plate are

$$
\mathbf{E}_{2 f}^{\prime}=A \sin \left(\frac{3 \pi}{4}\right) \exp \left[-i\left(\omega t-\mathbf{k} \cdot \mathbf{r}_{2}\right)\right] \cos \left(\frac{\pi}{4}\right)
$$




$$
\mathbf{E}_{2 s}{ }^{\prime}=A \sin \left(\frac{3 \pi}{4}\right) \exp \left[-i\left(\omega t-\mathbf{k} \cdot \mathbf{r}_{2}\right)\right] \sin \left(\frac{\pi}{4}\right)
$$

In above equations, $\mathbf{r}_{1}$ and $\mathbf{r}_{2}$ are the position vectors of light waves in arm 1 and arm 2, respectively. At the exit position of the interferometer, the light waves $\mathbf{E}_{1}{ }^{\prime}$ and $\mathbf{E}_{2}{ }^{\prime}$ will pass through the third quarter wave plate QWP 3 . Let $\mathbf{E}_{1}{ }^{\prime}$ become $\mathbf{E}_{1}$ after passing QWP 3. $\mathbf{E}_{1}$ can be decomposed into a vibration component $\mathbf{E}_{1 f}$ along the fast axis of QWP 3 and a vibration component $\mathbf{E}_{1 s}$ along the slow axis, which are expressed as

$$
\begin{gathered}
\mathbf{E}_{1 f}=\frac{1}{2} A \exp \left[-i\left(\omega t-\mathbf{k} \cdot \mathbf{r}_{1}\right)\right] \\
\mathbf{E}_{1 s}=-\frac{1}{2} A \exp \left[-i\left(\omega t-\mathbf{k} \cdot \mathbf{r}_{1}\right)\right] \exp \left(-i \frac{\pi}{2}\right)
\end{gathered}
$$

It is clear from Eq.7 and Eq.8 that linearly polarized light becomes right-handed circularly polarized light after QWP 3. Similarly, the fast and slow axis components of $\mathbf{E}_{2}$ after QWP 3 are

$$
\begin{gathered}
\mathbf{E}_{2 f}=\frac{1}{2} A \exp \left[-i\left(\omega t-\mathbf{k} \cdot \mathbf{r}_{2}\right)\right] \\
\mathbf{E}_{2 s}=\frac{1}{2} A \exp \left[-i\left(\omega t-\mathbf{k} \cdot \mathbf{r}_{2}\right)\right] \exp \left(-i \frac{\pi}{2}\right)
\end{gathered}
$$

Linearly polarized light $\mathbf{E}_{2}{ }^{\prime}$ passes through QWP 3 and becomes left-handed circularly polarized light. Two circularly polarized lights will be superimposed, and the superimposed $\mathbf{E}_{f}$ and $\mathbf{E}_{s}$ in the fast and slow axis directions of QWP 3 are

$$
\begin{aligned}
\mathbf{E}_{f} & =\mathbf{E}_{1 f}+\mathbf{E}_{2 f} \\
& =-A \cos \left[\frac{1}{2} \mathbf{k} \cdot\left(\mathbf{r}_{1}-\mathbf{r}_{2}\right)\right] \exp \left[-i\left(\omega t-\mathbf{k} \cdot \frac{\mathbf{r}_{1}+\mathbf{r}_{2}}{2}\right)\right] \\
\mathbf{E}_{s} & =\mathbf{E}_{1 s}+\mathbf{E}_{2 s} \\
& =-A \sin \left[\frac{1}{2} \mathbf{k} \cdot\left(\mathbf{r}_{1}-\mathbf{r}_{2}\right)\right] \exp \left[-i\left(\omega t-\mathbf{k} \cdot \frac{\mathbf{r}_{1}+\mathbf{r}_{2}}{2}\right)\right]
\end{aligned}
$$

From Eq.11 and Eq.12, it can be seen that two circularly polarized lights are superimposed into linearly polarized light. After that, the linearly polarized light is divided into four beams with equal intensities by the pyramid prism, and after passing through the imaging lens, they respectively pass through four polarization units with different polarization directions on the polarization array as shown in Fig.1. Define the angles of the polarization directions of the four polarization units to the fast axis of QWP 3 as $\psi_{i}(i=1,2,3,4)$, then the light vector after passing through each unit of the polarization array is

$$
\begin{aligned}
\mathbf{E}_{i}^{\text {out }} & =\mathbf{E}_{f} \cos \psi_{i}+\mathbf{E}_{s} \sin \psi_{i} \\
& =-A \cos \left[\frac{1}{2} \mathbf{k} \cdot\left(\mathbf{r}_{1}-\mathbf{r}_{2}\right)-\psi_{i}\right] \\
& \cdot \exp \left[-i\left(\omega t-\mathbf{k} \cdot \frac{\mathbf{r}_{1}+\mathbf{r}_{2}}{2}\right)\right]
\end{aligned}
$$

Finally, the intensities measured by CCD can be expressed as

$$
I_{i}=\mathbf{E}_{i}^{\text {out }} \cdot \mathbf{E}_{i}^{\text {out* }}=A^{2} \cos ^{2}\left[\frac{1}{2} \mathbf{k} \cdot\left(\mathbf{r}_{1}-\mathbf{r}_{2}\right)-\psi_{i}\right]
$$

where $\mathbf{k} \cdot\left(\mathbf{r}_{1}-\mathbf{r}_{2}\right)=2 \pi \sigma \Delta_{0} . \Delta_{0}$ is the optical path difference between the two arms of the interferometer. Assuming the total incident intensity is $I_{0}$, half will lost when passing the polarizer.
Then, Eq.14 becomes

$$
I_{i}=\frac{I_{0}}{4}\left[1+\cos \left(2 \pi \sigma \Delta_{0}-2 \psi_{i}\right)\right]
$$

Considering that the actual detection spectral line is Gaussian, the interference fringe visibility $V$ must be introduced into the interferogram. Meanwhile, by implementing the Doppler shift $\sigma=\sigma_{0}(1+v / c)$, the intensities can be write as follow

$$
\begin{aligned}
I_{i} & =\frac{I_{0}}{4}\left[1+V \cos \left(2 \pi \sigma_{0} \Delta_{0}+2 \pi \sigma_{0} \Delta_{0} \frac{v}{c}-2 \psi_{i}\right)\right] \\
& =\frac{I_{0}}{4}\left[1+V \cos \left(\varphi_{0}+\varphi_{w}-2 \psi_{i}\right)\right]
\end{aligned}
$$

It is known that the polarization directions of the polarization units $1,2,3$, and 4 in the polarization array are $0^{\circ}, 45^{\circ}, 90^{\circ}$, and $135^{\circ}$, respectively. Finally, four interferogram with different phases will be obtained on the detector simultaneously

$$
\begin{aligned}
& I_{1}=\frac{I_{0}}{4}(1+V \cos \Psi) \\
& I_{2}=\frac{I_{0}}{4}(1+V \sin \Psi) \\
& I_{3}=\frac{I_{0}}{4}(1-V \cos \Psi) \\
& I_{4}=\frac{I_{0}}{4}(1-V \sin \Psi)
\end{aligned}
$$

where $\Psi=\varphi_{0}+\varphi_{w}$. By solving Eq.17 to Eq.20, we can get

$$
\begin{gathered}
I_{0}=2\left(I_{1}+I_{3}\right)=2\left(I_{2}+I_{4}\right) \\
V=\frac{2\left[\left(I_{1}-I_{3}\right)^{2}+\left(I_{2}-I_{4}\right)^{2}\right]^{\frac{1}{2}}}{I_{0}} \\
\Psi=\arctan \left(\frac{I_{4}-I_{2}}{I_{3}-I_{1}}\right)
\end{gathered}
$$

Thus, the wind velocity and temperature of the target atmosphere can be obtained as

$$
\begin{gathered}
v=\frac{c}{2 \pi \sigma_{0} \Delta_{0}}\left(\Psi-\varphi_{0}\right) \\
T=-\frac{1}{Q \Delta_{0}^{2}} \ln V
\end{gathered}
$$

where $Q=2 \pi^{2} k \sigma^{2} /\left(m c^{2}\right), k$ is the Boltzmann constant and $m$ is the molecular weight. Therefore, by obtaining four interferograms from the system detector at the same time, extracting four intensity values and fixing the reference optical path difference of the interferometer system through instrument design and calibration, the wind velocity and temperature of the target atmosphere can be obtained.

\section{FIELD WIDENING DESIGN}

Because the prism splits the interference beam into quarters, the intensity of each interferogram is reduced to $1 / 4$, which means that the throughput of the system is also reduced to $1 / 4$. When the aperture and spectral resolution are constant, the throughput of the interferometer is completely determined by the incident intensity. The throughput of the system can be increased by increasing the amount of incident light if the optical train has been perfectly aligned. Field widening is one of the effective 
measures that can be taken. The target source for passive detection of the atmospheric wind field is faint airglow or aurora. In order to collect more light energy in the measurement, the interferometer is usually designed to allow oblique incidence at a relative large angle with a small change in optical path difference, which is called field widening. Field widening is usually achieved by adding glasses of different thicknesses and refractive indices to the two arms of the Michelson interferometer (Thuillier, Shepherd, 1985).

As is shown in Fig.1, we designed a field widening scheme using two glasses and a gap. According to the principle of Michelson interferometer, the original optical path difference is

$$
\begin{aligned}
\Delta \approx 2 & \left(n_{1} d_{1}+n_{2} d_{2}+n_{3} d_{3}\right) \\
& -\sin ^{2} i_{0}\left(\frac{d_{1}}{n_{1}}+\frac{d_{2}}{n_{2}}+\frac{d_{3}}{n_{3}}\right) \\
& -\frac{\sin ^{4} i_{0}}{4}\left(\frac{d_{1}}{n_{1}^{3}}+\frac{d_{2}}{n_{2}^{3}}+\frac{d_{3}}{n_{3}^{3}}\right)
\end{aligned}
$$

where $i_{0}$ is the angle of the light beam incident into the beamsplitter, $d_{1}, d_{2}, d_{3}, n_{1}, n_{2}, n_{3}$ are the thicknesses and refractive indices of glass 1, glass 2 and the air gap, respectively. To realize the field widening of the system, the reference optical path difference should be non-sensitive to the field of view, which means the high order of Eq.26 should equal to zero. Generally, there are three requirements for field widening and thermal compensation: the optical path difference does not vary with the incident angle; the optical path difference does not vary with the incident wavelength; the optical path difference does not vary with temperature (Thuillier, Hersé, 1991). The field widening requirements can be expressed as

$$
\begin{gathered}
\frac{d_{1}}{n_{1}}+\frac{d_{2}}{n_{2}}+\frac{d_{3}}{n_{3}}=0 \\
\frac{d_{1}}{n_{1}^{2}} \frac{\partial n_{1}}{\partial \lambda}+\frac{d_{2}}{n_{2}^{2}} \frac{\partial n_{2}}{\partial \lambda}+\frac{d_{3}}{n_{3}^{2}} \frac{\partial n_{3}}{\partial \lambda}=0 \\
d_{1}\left(n_{1} \alpha_{1}+\beta_{1}\right)+d_{2}\left(n_{2} \alpha_{2}+\beta_{2}\right)+d_{3}\left(n_{3} \alpha_{3}+\beta_{3}\right)=0
\end{gathered}
$$

where $\alpha_{i}(i=1,2,3)$ is the thermal expansion coefficient of each layer and $\beta_{i}$ is the thermal coefficient of the refractive index.

According to the field of view compensation principle, when designing a field widened interferometer, two pieces of compensation glass and an air gap are usually used to achieve the field widening. We give a compensation scheme for the NSPWII system with the reference optical path difference of $\Delta_{0}=4.5 \mathrm{~cm}$, which is shown in Table 1. Detailed data of glass LF5 and N-LaF21 can be found in the glass database provided by SCHOTT, Germany (SCHOTT, 2018). According to the de-

\begin{tabular}{ccc}
\hline Name & Material & Thickness(cm) \\
\hline Glass 1 & LF5 & 6.07636 \\
Glass 2 & N-LaF21 & 6.52867 \\
Air gap & none & 0.19100 \\
\hline
\end{tabular}

Table 1. NSPWII system compensation glass design scheme

signed scheme of compensation glass in Table 1, the deviation of the reference optical path difference with the incident angle under the incidence of different wavelengths is obtained using the relevant glass parameters, which is shown in Fig.2. It can be seen that in the range of a $3^{\circ}$ field of view, the deviation of the reference optical path difference of the three wavelengths can be controlled within $0.00005 \mathrm{~cm}$. Comparing with the reference optical path difference of $4.5 \mathrm{~cm}$, it can be considered that the field widening and achromatic compensation are achieved. Figure 3 shows the variation of $d \Delta_{0} / d T$ with temperature. The variation can be controlled on the order of $10^{-6} \mathrm{~cm} / \mathrm{K}$ (at $20^{\circ}$ C), and a good thermal compensation is obtained.

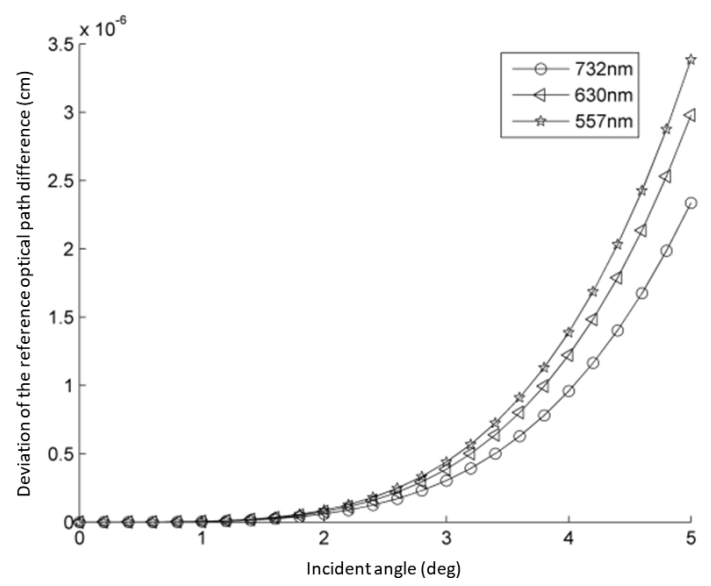

Figure 2. The deviation of the reference optical path difference with the incident angle

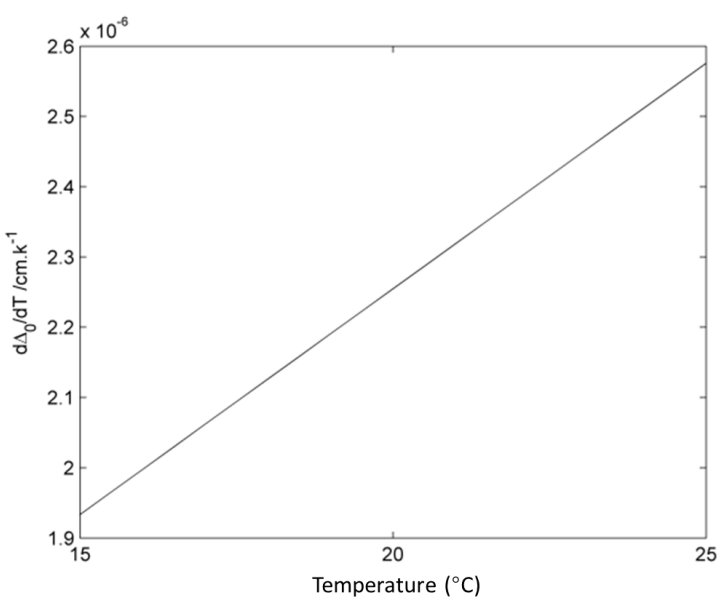

Figure 3. The variation of $d \Delta_{0} / d T$ with temperature

Besides, the pyramid prism is one of the core devices in the NSPWII system. Its function is to divide a beam of light into four beams in order to achieve four-intensity simultaneous detection. The prism has a square bottom surface and four sides are symmetrical isosceles triangles. A beam of uniform incident light within a certain incident angle passes through the four sides, and is refracted by the sides and bottom of the pyramid prism. Thus, it can be divided into four beams to achieve beam splitting. However, in the design, the light should be separated outside the pyramid prism. If the light split before the bottom surface, the designed prism size will be very large, which is not suitable for the installation of the NSPWII system.

While designing a pyramid prism, there are usually two factors to consider: one is to maximize the energy of light passing through the prism, the other is to match the size of the pyramid prism with the imaging lens and CCD in the NSPWII system. Considering the above two factors, choosing a proper side length of the prism and the material of the prism is significant 
to the optimization design of the pyramid prism. In addition, quartz glass has good thermal stability, good light transmission performance, high temperature resistance, corrosion resistance, and good electrical insulation properties, which is a good choice for the pyramid prism.

\section{MODELING AND SIMULATION}

Based on the principle designed system scheme of NSPWII, we modeled the instrument in TracePro to verify its feasibility by computer simulation. The parameters of every component are defined in the software, including the size, material, refraction index, and polarization properties of the PBS, two glass arms and the pyramid prism. After defining the optical properties of the NSPWII system, the next step is to set the light source. According to the requirements of the NSPWII system, a surface light source was used here. The emitting angle of the light at each point of the surface light source is controlled within a range of $3^{\circ}$. Then the ray tracing has been done.

We analyzed the system using the results from ray tracing. Several detector surfaces were inserted into different parts of the instrument to obtain the polarization states of the light when passing through a specific component. First, we analyzed the polarization states of the light emitted from the arm 1 and arm 2 after passing through the third quarter wave plate, as shown in Fig. 4 and Fig. 5, respectively. It can be seen that the light waves emitted from the arm 1 and arm 2 become right-handed and left-handed circularly polarized light after QWP 3, respectively, which is consistent with the results of theoretical analysis. Second, we analyzed the polarization state of the light wave after passing through the polarization array, which is shown in Fig. 6. Finally, we analyzed the light intensity distribution obtained on the CCD. Figure 7 shows the light flux distribution on CCD obtained by optical simulation. It can be seen that the light beam is divided into four beams by the pyramid prism, and four interferograms with different intensities formed on the $\mathrm{CCD}$ at the same time after passing through the imaging lens, which preliminary verified that the optical structure of the NSPWII system is feasible.

The images of the field of view are not shown here because we didn't add the fore-optics nor the field stop in the system simulation. In the simulation, we verified the feasibility of the field widened polarization Michelson interferometer, which is the core component of the NSPWII. In practice, there are still many detailed problems need to be analyzed. For a totally solid system, we can replace the air gap with a third glass slab. The PBS and the glass slabs are cemented together, and the quarter wave plates and reflecting mirror can be evaporated on the glass slabs. These techniques provide a more static, compact NSPWII instrument.

\section{CONCLUSION}

In conclusion, we proposed a theoretical model and design of the NSPWII. The incident light pass through a group of polarizers and waveplates, a field widened Michelson interferometer. Then it is split by a pyramid prism, and modulated by a polarization array. Based on the principle of polarization interference, four interferograms with different phases on the four partitions of the detector can be obtained simultaneously. Therefore, NSPWII can realize real time detection of targets, and the defect that the moving mirror scanning mode cannot accurately
Polarization Map for Incident Flux OWP3 Surface 2

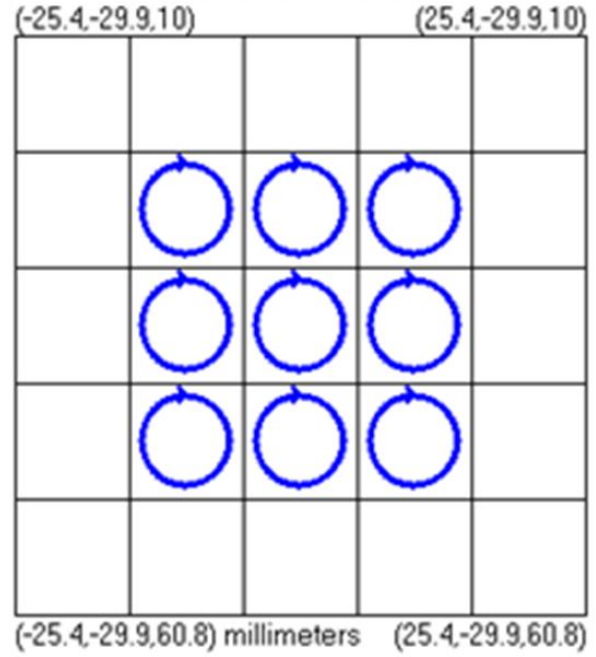

Figure 4. The polarization state of the light wave emitted from arm 1 after passing through QWP 3

Polarization Map for Incident Flux OWP3 Surface 2

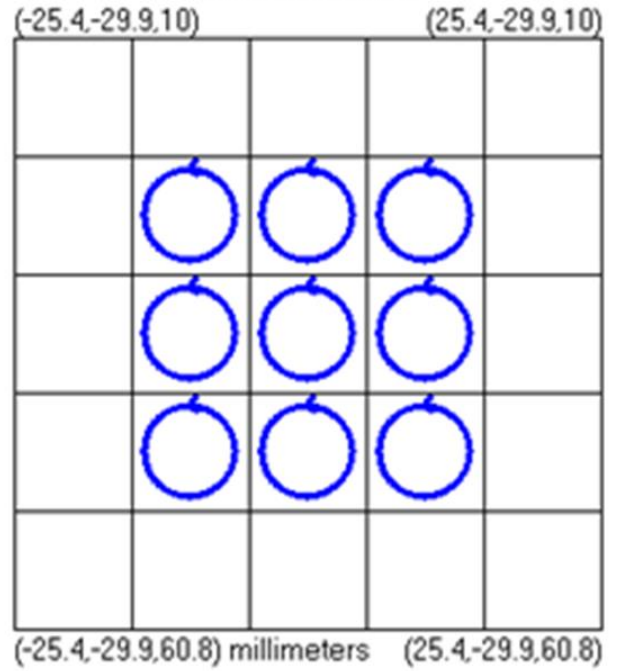

Figure 5. The polarization state of the light wave emitted from arm 2 after passing through QWP 3

detect fast-changing targets has been overcome. The advantages of this instrument are static (no moving parts), achromatic, and temperature-compensated. It is capable of measuring the upper atmospheric wind field in real time with a high precision. The field widening design is demonstrated and the simulation is conducted. The feasibility of the system is verified. Further research will focus on the optimization and the error analysis of the system.

\section{ACKNOWLEDGEMENTS}

The work was supported by Major Program of the National Natural Science Foundation of China (41530422), The 863 Program (2012AA121101), National Natural Science Foundation of China $(61775176,61405153,61501361)$. 
Polarization Map for Incident Flux cod Surface 4

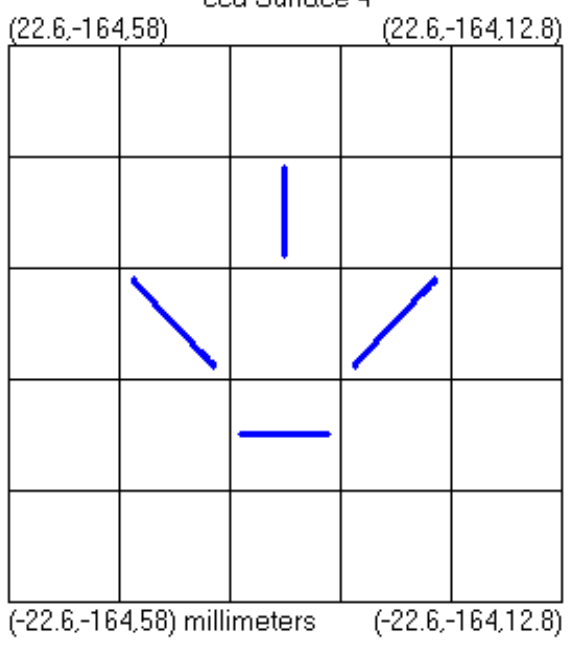

Figure 6. Diagram of the polarization state of the light wave after passing through a polarization array
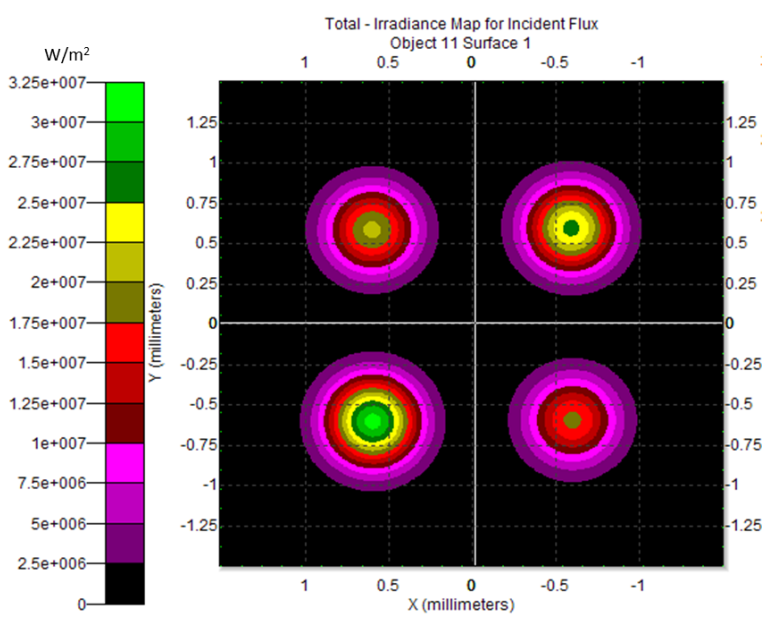

Figure 7. Light flux distribution on the CCD in optical simulation

\section{REFERENCES}

Babcock, D. D., 2006. Mesospheric imaging Michelson interferometer instrument development and observations. $\mathrm{PhD}$ thesis, York University.

Bird, J. C., Liang, F., Solheim, B. H., Shepherd, G. G., 1995. A polarizing Michelson interferometer for measuring thermospheric winds. Measurement Science and Technology, 6(9), 1368-1378. https://doi.org/10.1088

Gault, W. A., McDade, I. C., Shepherd, G. G., Mani, R., Brown, S., Gregory, P., Scott, A., Rochon, Y. J., Evans, W. F., 2001. Swift: an infrared doppler michelson interferometer for measuring stratospheric winds. Sensors, Systems, and NextGeneration Satellites V, 4540, International Society for Optics and Photonics, 476-481.

Kristoffersen, S., Ward, W., Brown, S., Drummond, J., 2013. Calibration and validation of the advanced E-Region Wind
Interferometer. Atmospheric Measurement Techniques, 6(7), 1761-1776.

Langille, J. A., Ward, W. E., Scott, A., Arsenault, D. L., 2013. Measurement of two-dimensional Doppler wind fields using a field widened Michelson interferometer. Applied optics, 52(8), 1617-1628.

Ramsey, H. et al., 1980. Improvements in birefringent filters. 6: analog birefringent elements. Applied Optics, 19(12), 20462058 .

SCHOTT, 2018. Optical Glass 2018. Advanced Optics SCHOTT AG.

Shepherd, G. G., 2002. Spectral imaging of the atmosphere. 82, Academic press.

Shepherd, G. G., Gault, W. A., Miller, D., Pasturczyk, Z., Johnston, S. F., Kosteniuk, P., Haslett, J., Kendall, D. J., Wimperis, J., 1985. WAMDII: wide-angle Michelson Doppler imaging interferometer for Spacelab. Applied optics, 24(11), 1571-1584.

Shepherd, G. G., Thuillier, G., Cho, Y.-M., Duboin, M.-L., Evans, W. F., Gault, W., Hersom, C., Kendall, D., Lathuillere, C., Lowe, R. et al., 2012. The wind imaging interferometer (WINDII) on the upper atmosphere research satellite: a 20 year perspective. Reviews of Geophysics, 50(2).

Thuillier, G., Hersé, M., 1991. Thermally stable field compensated Michelson interferometer for measurement of temperature and wind of the planetary atmospheres. Applied optics, 30(10), 1210-1220.

Thuillier, G., Shepherd, G. G., 1985. Fully compensated Michelson interferometer of fixed-path difference. Applied optics, 24(11), 1599-1603. 